Wolf-Rayet Phenomena in Massive Stars and Starburst Galaxies

Proceedings IAU Symposium No. 193, (c) 1999 IAU

K.A. van der Hucht, G. Koenigsberger \& P.R.J. Eenens, eds.

\title{
Spectral analyses of WC stars in the LMC
}

\author{
Götz Gräfener, Wolf-Rainer Hamann, and Lars Koesterke \\ Department of Physics, University of Potsdam, Germany
}

\begin{abstract}
Spectra of seven WC stars in the LMC are taken with the HSTFOS. They are analyzed by means of non-LTE models for spherically expanding atmospheres with complex model atoms of helium, carbon and oxygen taken into account. We find stellar luminosities in the range from $10^{5.1-5.6} \mathrm{~L}_{\odot}$. The stellar temperature $T_{\star}$, defined as the effective temperature related to the stellar core radius, is of the order of $100 \mathrm{kK}$. The atmospheric compositions show carbon mass fractions of about 0.4 and oxygen mass fractions in the range $0.1-0.5$.
\end{abstract}

\section{Analyses}

In the observed sample all single WC and WO stars from the catalog of Breysacher (1981) are considered. Due to the known distance and low interstellar extinction of the LMC, reliable stellar parameters can be obtained by spectral analyses. The results are presented in Table 1. A detailed description of the observations and methods of analysis is given in Gräfener et al. (1998).

Table 1. Model parameters: stellar luminosity $L_{\star}$; core radius $R_{\star}$; effective core temperature $T_{\star}$; mass loss rate $\dot{M}$; terminal velocity $v_{\infty}$; surface mass fraction of carbon $\beta_{\mathrm{C}}$ and oxygen $\beta_{\mathrm{O}}$.

\begin{tabular}{lcccrcrcc}
\hline star & type & $\begin{array}{c}\log L_{\star} \\
\left(\mathrm{L}_{\odot}\right)\end{array}$ & $\begin{array}{c}R_{\star} \\
\left(\mathrm{R}_{\odot}\right)\end{array}$ & $\begin{array}{r}T_{\star} \\
(\mathrm{kK})\end{array}$ & $\begin{array}{c}\log \dot{M} \\
\left(\mathrm{M}_{\odot} \mathrm{yr}^{-1}\right)\end{array}$ & $\begin{array}{c}v_{\infty} \\
\left(\mathrm{km} \mathrm{s}^{-1}\right)\end{array}$ & $\beta_{\mathrm{C}}$ & $\beta_{\mathrm{O}}$ \\
\hline Br 7 & WC 4 & 5.29 & 1.65 & 94.6 & -4.16 & 2300 & 0.4 & 0.2 \\
Br 8 & WC 4 & 5.13 & 1.13 & 104.2 & -4.06 & 2300 & 0.4 & 0.3 \\
Br 10 & WC 4 & 5.62 & 2.52 & 92.5 & -3.80 & 2800 & 0.5 & 0.2 \\
Br 43 & WC 4 & 5.26 & 1.32 & 103.9 & -3.91 & 2600 & 0.4 & 0.3 \\
Br 50 & WC 4 & 5.55 & 2.25 & 94.0 & -3.72 & 2800 & 0.4 & 0.1 \\
Br 74 & WC 4 & 5.13 & 1.13 & 104.2 & -4.06 & 2300 & 0.4 & 0.3 \\
Br 93 & WO 4 & 5.10 & 1.17 & 101.0 & -4.41 & 3600 & 0.4 & 0.5 \\
\hline
\end{tabular}



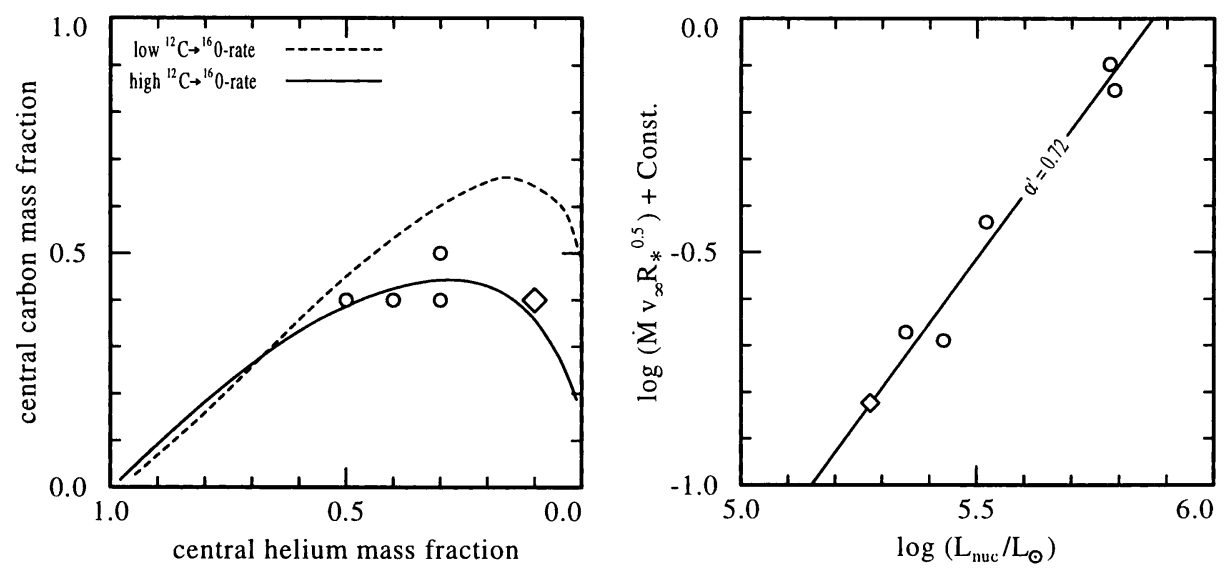

Figure 1. Left: Surface abundances are compared to central abundances of core helium burning stars with different values of the ${ }^{12} \mathrm{C}(\alpha, \gamma){ }^{16} \mathrm{O}$ rate; Right: Mass-loss rates are compared to a wind momentum-luminosity relation with a force multiplier of $\alpha^{\prime}=0.72$. The luminosities $L_{\text {nuc }}$ are corrected for the flux of kinetic wind energy.

\section{Surface abundances, mass-loss rates}

Since for massive stars the central temperature in the helium burning core is nearly independent of the stellar mass, there exists a relation between central helium and carbon mass fractions. The shape of this relation - in particular the maximum value for carbon - mainly depends on the ratio of the ${ }^{12} \mathrm{C}+\alpha \rightarrow{ }^{16} \mathrm{O}$ rate to the process $3 \alpha \rightarrow{ }^{12} \mathrm{C}$. Because of their large mass-loss rates WC stars reveal layers at their surface which have been part of a convective helium burning core and show the corresponding abundances. As is shown in Figure 1 the derived abundances are in good agreement with the high ${ }^{12} \mathrm{C}+\alpha \rightarrow{ }^{16} \mathrm{O}$ rate from Caughlan et al. (1985).

The right panel of Figure 1 shows that the WC mass-loss rates obey a wind momentum-luminosity relation analogous to the one which was derived by Puls et al. (1996) from the theory of line-driven winds for $\mathrm{O}$ stars. The fact that our homogeneous sample of WR stars obeys such a relation over a wide range of luminosities is a hint to the line-force as driving mechanism for WR winds.

\section{References}

Breysacher, J. 1981, A\&AS 43, 203

Caughlan, G.R., Fowler, W.A., Harris, M.J., Zimmermann, B.A. 1985, Atomic Data Nuc. Data Tables 32, 197

Gräfener, G., Hamann, W.-R., Hillier, D.J., Koesterke, L. 1998, A\&A 329, 190

Puls, J., Kudritzki, R.-P., Herrero, A., Pauldrach, A.W.A., Haser, S.M., Lennon, D.J., Gabler, R., Voels, S.A., Vílchez, J.M., Wachter, S., Feldmeier, A. 1996, A\&A 305, 171 